\title{
Method for digitizing paper archive of solar radio observations made with Large Pulkovo Radio Telescope
}

\author{
Vladimir E. Abramov-Maximov \\ Central Astronomical Observatory at Pulkovo, St.Petersburg, 196140, Russia \\ email: beam@gao.spb.ru
}

\begin{abstract}
Valuable large archive of solar radio observations has been accumulated by the Pulkovo solar radio group. But big part of data is recorded on paper. We describe a method and software for transferring data recorded on paper to FITS format.
\end{abstract}

\section{Introduction}

Pulkovo solar radio group has accumulated a big archive of solar microwave observations using Large Pulkovo Radio Telescope (Khaikin et al. (1960)). This archive contains the data for about three solar cycles. These data is a uniform series of daily observations. The summary of the archive is given in Abramov-Maksimov et al. (1999). But the base part (till 1998) of data is presented on paper medium and has not been analyzed yet. So it is important to transfer data from paper to digital form.

\section{Observational material}

The result of one observation is a set of one-dimensional scans on some fixed wavelengths $(2.0,2.3,2.7,3.2,4.4,6.6,9$ and $20 \mathrm{~cm})$. Number of wavelengths varied from 3 to 7 in different periods of observations. Two polarizational parameters (intensity $I$ and circular polarization $V$ ) are recorded. There are two types of traces. In the first type intensity and circular polarization are recorded in different traces. In the second type intensity and circular polarization are recorded in one trace with a bit shift in time scale. As a rule in this case different colors for intensity and circular polarization was used. The length of trace of one scan varies from $40 \mathrm{~cm}$ to $140 \mathrm{~cm}$ in different period of observations. The height of trace is about $30 \mathrm{~cm}$. So each trace can be split up into several A4 parts (frames) for scanning. The total number of scans is about 60000.

\section{Stages of processing}

We distinguish the following steps in the processing

- preparation of the material for scanning;

- scanning;

- creating and cataloging CD archive of images;

- digitizing images;

- editing (if it is necessary);

- rotating (if the image is turned);

- combining frames;

- making FITS-file. 


\section{Processing}

\subsection{Preparation of the material for scanning and scanning}

We use scanners of format A4 (HP ScanJet 5P and HP ScanJet 7400). Each trace is split up on some frames for scanning. To combine parts of scan from adjacent frames the operator put marks on upper corners of each frames manually. These marks must be present in adjacent frames. We scan images with resolution 200 dpi in 256 color mode. Images are saved in BMP-format.

\subsection{Cataloging $C D$ archive of images}

Now CD archive of images embraces about of $170 \mathrm{CD}$. To manage this archive we develop software which scans CDs, create catalogs of CDs, sort catalogs and creates combined catalog of scanned images. So it is easy to get the name of CD by date and wavelength.

\subsection{Digitizing, rotating and combining frames}

Digitizing of graph from scanned image is not a problem. The problem is to digitize a bulk of data during short time.

First of all, we tried some programs which can be downloaded from Internet (Grafula, Tracer, Graph Digitizer, SMGraph, Ciphering, Graph2Digit, GetData). We tried to use IDL also to solve this problem (Grechnev et al. (2004)). All these programs and methods can solve our problem. But the unit of processing of these programs is one image (file or scan). And programs work in interactive mode only and require a much time for digitizing. So these programs are useful to digitize a bit data, but it is difficult to process a bulk of data.

We decided to use another method. The unit of processing is the set of scans (for week, month or year - it depends on free disk space). And process of digitizing, rotating and combining frames includes some iterations in batch mode. The operator prepares a task for processing, then starts the task. After finishing the task operator looks results, corrects the task and runs it once more. And so on. The program of digitizing analyzes pixels value (colors) and count of pixels of different colors on each image. Colors and numbers of pixels of scans, grid and background are differed. This fact is used to find pixels of scans.

\section{Conclusions}

We have developed method and software to digitize graphical data recorded on paper medium. Our method can be apply to digitize large paper archives, for example, archive of solar radio observations using Large Pulkovo Radio Telescope.

\section{Acknowledgements}

This work was supported by the RFBR (grants 02-02-16548, 03-02-17357), Dogovor 16KI, Dogovor OFN18 and Dogovor of Prezidium of RAS 8/2004.

\section{References}

Abramov-Maksimov, V. E., Bogod, V. M., Borisevitch, T. P., Korzhavin, A. N., Opeikina, L. V. \& Peterova, N.G. 1999 In Proc. of the VIII Russian-Finnish Symposium on Radioastronomy, June 28-July 3, Saint Petersburg, 108-111.

Khaikin, S. Eh., Kajdanovskij, N. L., Esepkina, N. A. \& Shivris, O. N. 1960, Izv. GAO USSR $A S, \mathbf{1 6 4}, 3-26$.

Grechnev, V. V., Abramov-Maksimov, V. E., Peterova, N. G., Borisevich, T. P., Kaltman, T. I. \& Meshalkina, N. S. 2004 Izv. SAO RAS, in press. 